\title{
The Perception of Health and Cyberbullying Sensitivity in Adolescents
}

\author{
Ayla Hendekci ${ }^{1}$, Türkan Kadiroğlu ${ }^{2}$ \\ ${ }^{1}$ Giresun University, Faculty of Health Sciences, Department of Public Health Nursing, Giresun, Turkey \\ ${ }^{2}$ Atatürk University, Faculty of Nursing, Department of Child Health Nursing, Erzurum, Turkey \\ Received: 06 November 2019, Accepted: 13 April 2020, Published online: 30 April 2020 \\ C Ordu University Institute of Health Sciences, Turkey, 2020
}

\begin{abstract}
Objective: This study aims to determine the relationship between adolescents' perceptions of health and their susceptibility to cyberbullying and the factors that affect them.

Method: The targeted population of this descriptive study is the students of middle schools managed by the Directorate of National Education in a province in the Black Sea region. The selection of subjects was made by a simple random sampling method from two determined middle schools in the city center $(n=381)$. The data of the study were collected with the Introductory Information Form, the Perception of Health Scale and the Cyberbullying Sensitivity Scale. The face-to-face interview method was used in data collection. The research was conducted according to ethical principles.

Results: Of the adolescents who participated in the study, 55.4\% were girls and $72.7 \%$ lived in a nuclear family and most of the parents' educational level was high school graduated. There is a significant difference between the cyberbullying sensitivity scale score averages according to the gender of adolescents $(\mathrm{p}<0.05)$. There was a negatively significant association between the precision sub-dimension of the perception of health scale and the cyberbullying sensitivity scale for adolescents $(\mathrm{p}<0.05)$.

Conclusion: The study found that both male and female adolescents had a high level of cyberbullying sensitivity, good health perceptions, higher cyberbullying sensitivity of female students and a negative relationship between the 'precision' sub-dimension of health perception and the cyberbullying sensitivity. It is suggested that the awareness of adolescents should be raised about their perception of good health and similar studies should be repeated.
\end{abstract}

Key words: Adolescent, Perception of Health, Cyberbullying

Suggested Citation: Hendekci A, Kadiroglu T. The Perception of Health and Cyberbullying Sensitivity in Adolescents. Middle Black Sea Journal of Health Science, 2020; 6(1):18-23.

\section{Address for correspondence/reprints:}

Ayla Hendekci

Telephone number: +90 (539) 3570628

ORCID-ID 0000-0001-7974-6232

E-mail: ayla.hendekci@gmail.com

DOI: $10.19127 /$ mbsjohs.643779

\section{Introduction}

The perception of health is a combination of an individual's personal feelings, thoughts, prejudices, and expectations regarding his/her own health (Velderman et al., 2010). Having positive beliefs about the individual's own health is defined as "the perception of good health" while having negative beliefs is defined as "the perception of bad health". The perception of good health aims to ensure that people acquire and sustain life behaviors that improve 
health (Velderman et al., 2010; Bademli and Lok, 2018).

The perception of health in the adolescent period, which has its own characteristics and is complex, is decisive for the following years. Adolescents may adopt patterns of behavior that improve their health or gain unhealthy lifestyle behaviors that would harm both themselves and others (WHO, 2016). Cyberbullying is one of the conditions that has become increasingly common during the adolescent periods, considered a major public health issue, and thought to cause unhealthy lifestyle behaviors (Baldry et al., 2019).

Cyberbullying is the use of information and communication technologies by an individual or group maliciously and repetitively with the intent to harm other individuals (Katz et al., 2019). Adolescents are the most exposed ones to the act of cyberbullying (Baldry et al., 2019; Katz et al., 2019). Studies with adolescents in Turkey have found that cyberbullying is at a fairly high level (Akbulut and Eristi, 2011; Yaman and Sonmez, 2015; Kilinç and Gunduz, 2017; Uludasdemir and Kucuk, 2019).

Studies have shown that sociodemographic characteristics, media, school, and psychological factors can also affect cyberbullying, hence, cyberbullying sensitivity (Shin and Ahn, 2015; Olenik-Shemesh and Heiman, 2017; Al-Rahmi et al., 2018), and the perception of health is thought to be one of these variables. We encounter no studies on adolescents' health perceptions and sensitivities to cyberbullying in the literature. For this reason, this study was conducted to determine the relationship between adolescents' health perceptions and sensitivities to cyberbullying.

\section{Methods}

\section{Study Aim and Design}

The design of this study is descriptivecorrelational. This study investigated the relationship between the health perceptions of adolescents between the ages of 12 and 15 and their sensitivity to cyberbullying and the factors affecting them. The criteria for participation in the survey were to be willing to participate in the survey and to be in the mentioned age range.

\section{Participants}

The population of this study consists of the 5, 6 and 7th-grade students of twenty middle schools in a province in the Black Sea region. In the study, the sample size was determined as 370 with 95\% confidence interval by the known population sampling method. To ensure equal representation of the population, two secondary schools from all socioeconomic levels (low, medium, high) were determined by a simple random sampling method. The classes to be sampled in 5, 6 and 7th grades of the determined schools were also determined using a simple random sampling method. Written and oral consent was obtained from the parents and students who were studying in these classes and voluntarily wanted to participate in the research. The study was completed with 381 adolescents after excluded 43 students who did not agree to participate in the research and 18 students who did not come to school or were on leave at the date of data collection $(n=$ 381).

\section{Data Collection}

In this research, the Introductory Information Form, Perception of Health Scale (PHS) and Cyberbullying sensitivity scale were used as data collection tools. The face-to-face interview method was used as a method of data collection. The questionnaire was filled out in the class and it took approximately 25-30 minutes to complete each form.

Introductory Information Form: This form, prepared by the researchers, consists of 7 questions about sociodemographic characteristics such as age, gender, family type, and health status and social activity

Perception of Health Scale (PHS); The scale developed by Diamond et al (2007) was translated into Turkish by Kadioglu and Yildiz (2012). PHS is a five-point Likert scale consisting of 15 items and four sub-factors. The item numbers of sub-dimensions of the scale are as follows; The control center, 1,2,3,4,5; Precision, 6,7,8,9; Importance of health, 10,11,12; Self-awareness, 13,14,15. For the scale, positive statements are rated as "very agreeable $=5$ ", "I agree= 4", "I am undecided= 3", "Disagree= 2", "I do not agree at all. Also, 2, 3, 4, 6, 7, 8, 12, 13rd and 15th articles are negative expressions. Negative expressions were reverse scored. The minimum score on the scale is 15 and the maximum score is 75 . The Cronbach alpha value of the scale is 0.82 (Diamond et al., 2007; Kadioglu and Yildiz, 2012). In this study, the scale Cronbach alpha value was found to be 0.51 .

Cyberbullying Sensitivity Scale (CBSS); The scale developed by Tanrikulu et al. (2013) consists of 14 items and is answered on a triple scale (Yes, Sometimes, No). Questions on the scale are evaluated as "Yes"-3 points, "Sometimes"-2 points, "No"-1 
point. The scale has a single factor structure, and the highest score from the scale is 42 , and the lowest score is 14 . The scale Cronbach alpha value is 0.87 (Tanrikulu et al., 2013). In this study, the Cronbach alpha value of scale was found to be 0.82 .

\section{Data Analysis}

IBM SPSS Statistics for Windows, (Version 20.0; Armonk, New York) statistical package software was used to evaluate research data. Number, percentage, arithmetic mean, standard deviation and median (min, max) values were given for the descriptive properties of the data. Shapiro-Wilk normality test was used to see if the data showed normal distribution. The data did not show a normal distribution. Therefore, Mann Whitney U, Kruskal Wallis $\mathrm{H}$, and Spearman nonparametric correlation tests were performed to statistical tests for hypothesis testing and their assumptions. The level of statistical significance was accepted as $\mathrm{p}<0.05$.

\section{Ethical Consideration}

For the research, approval from the ethics committee of a university (Protocol Number: 20194/8 Date:10.07.2019), the written permission from the relevant institutions, and the oral and written consent from the students and their families who participated in the research were obtained.

\section{Results}

Looking at the characteristics of adolescents, $55.4 \%$ of the participants were female students, $72.7 \%$ had the nuclear family, high school was the most common level of education as $36.7 \%$ in the mother's education, and as $40.7 \%$ in the father's education, also, $87.7 \%$ of the participants expressed themselves as healthy and $53.5 \%$ of those used to involve in a social activity. When the sensitivity levels of cyberbullying according to the characteristics of adolescents were examined, the difference between the score averages of adolescents from the cyberbullying sensitivity scale was found to be statistically significant according to their gender. $(\mathrm{p} \leq 0.001)$ (Table 1).

Table 1. The Levels of Cyberbullying Sensitivity by Descriptive Characteristics of Adolescents $(n=381)$

\begin{tabular}{|c|c|c|c|c|}
\hline \multicolumn{5}{|l|}{ Age Mean \pm SD $13.25 \pm 1.187$} \\
\hline Descriptive Characteristics & $\mathbf{n}$ & $\%$ & Median (Min-Max) & Test and $p$ \\
\hline \multicolumn{5}{|l|}{ Gender } \\
\hline Female & 211 & 55.4 & $36.00(13.00-42.00)$ & \multirow{2}{*}{$\begin{array}{c}\mathrm{U}=14334.500 \\
\mathbf{p}=\mathbf{0 . 0 0 1}\end{array}$} \\
\hline Male & 170 & 44.6 & $34.00(21.00-39.00)$ & \\
\hline \multicolumn{5}{|l|}{ Family Type } \\
\hline Nuclear family & 277 & 72.7 & $36.00(13.00-42.00)$ & \multirow{3}{*}{$\begin{array}{c}\mathrm{KW}=.804 \\
\mathrm{p}=0.669\end{array}$} \\
\hline Extended family & 80 & 21.0 & $35.00(22.00-39.00)$ & \\
\hline Broken Family & 24 & 6.3 & $35.00(26.00-40.00)$ & \\
\hline \multicolumn{5}{|l|}{ Mother's Education Status } \\
\hline First-secondary education & 116 & 30.4 & $33.50(26.00-39.00)$ & \multirow{3}{*}{$\begin{array}{c}\mathrm{KW}=.651 \\
\mathrm{p}=0.722\end{array}$} \\
\hline High school & 140 & 36.7 & $36.00(29.00-39.00)$ & \\
\hline University and higher & 125 & 32.8 & $35.50(13.00-42.00)$ & \\
\hline \multicolumn{5}{|l|}{ Father's Educational Status } \\
\hline First-secondary education & 109 & 28.6 & $35.00(16.00-39.00)$ & \multirow{3}{*}{$\begin{array}{c}\mathrm{KW}=.699 \\
\mathrm{p}=0.705\end{array}$} \\
\hline High school & 155 & 40.7 & $35.00(21.00-40.00)$ & \\
\hline University and higher & 117 & 30.7 & $36.00(13.00-42.00)$ & \\
\hline \multicolumn{5}{|l|}{ Health situation } \\
\hline Healthy & 334 & 87.7 & $35.50(13.00-42.00)$ & \multirow{2}{*}{$\begin{array}{c}\mathrm{U}=6786.000 \\
\mathrm{p}=0.130\end{array}$} \\
\hline Not healthy & 47 & 12.3 & $34.00(21.00-39.00)$ & \\
\hline \multicolumn{5}{|l|}{ Social Activity } \\
\hline Involving & 204 & 53.5 & $36.00(13.00-40.00)$ & \multirow{2}{*}{$\begin{array}{c}U=17045.500 \\
p=0.344\end{array}$} \\
\hline Not involving & 177 & 46.5 & $35.00(16.00-42.00)$ & \\
\hline
\end{tabular}

The control center sub-dimension score average for the perception of health scale of adolescents is $16.62 \pm 2.45$, the precision sub-dimension score average is $12.67 \pm 2.75$, the importance of health subdimension score average is $10.29 \pm 2.16$, the selfawareness sub-dimension score average is $8.21 \pm 1.79$ and the total health perception score average is $47.81 \pm 4.79$ (Table 2). 
Table 2. The Perception of Health Score Averages of Adolescents $(n=381)$

\begin{tabular}{lcc}
\hline $\begin{array}{l}\text { The Perception of } \\
\text { Health Sub-dimensions }\end{array}$ & Mean \pm SD & Min-Max \\
\hline Control Center & $16.62 \pm 2.45$ & $7-21$ \\
Precision & $12.67 \pm 2.75$ & $4-20$ \\
The Importance of Health & $10.29 \pm 2.16$ & $3-15$ \\
Self-Awareness & $8.21 \pm 1.79$ & $3-14$ \\
Total & $47.81 \pm 4.79$ & $34-61$ \\
\hline
\end{tabular}

It was found that there was a negative correlation between the precision sub-dimension score averages of the adolescents' health perception scale and the cyberbullying sensitivity $(\mathrm{p}<0.05)$. When the relationship between the perception of health and cyberbullying sensitivity total scores was examined, it was not found a statistically significant correlation $(\mathrm{p}>0.05)$ (Table 3).

Table 3. The Relationship Between Adolescents' Perception of Health and Cyberbullying Sensitivities $(\mathrm{n}=381)$

\begin{tabular}{lcc}
\hline & \multicolumn{2}{c}{$\begin{array}{c}\text { The Sensitivity to } \\
\text { Cyberbullying }\end{array}$} \\
\hline $\begin{array}{l}\text { The Perception of } \\
\text { Health Sub-Dimensions }\end{array}$ & $\mathbf{r}$ & $\mathbf{p}$ \\
\hline Control Center & -.22 & 0.668 \\
Precision & -.116 & $\mathbf{0 . 0 2 4}$ \\
The Importance of & .079 & 0.122 \\
Health & .038 & 0.456 \\
Self-Awareness & .006 & 0.908 \\
Total Points & & \\
\hline
\end{tabular}

\section{Discussion}

The findings of the study, which aimed to determine the relationship between the health perceptions of adolescents in Turkey and their sensitivity to cyberbullying, were discussed in this section. The cyberbullying sensitivity score of adolescents participating in the study was 36.00 for female students and 34.00 for male students. Accordingly, it is observed that both male and female adolescents have a high level of sensitivity to cyberbullying. In a study of adolescents, the sensitivity score for cyberbullying was expressed as high as 33.04 for girls and 30.81 for boys (Bridge and Duman, 2019). In a study conducted to determine the cyberbullying sensitivity of teacher candidates, the scale score of the participants was stated as similarly high as 32.37 (Uysal et al., 2014). The high level of cyberbullying sensitivity in both male and female students may be thought to be due to increased bullying exposure. Some studies have shown that exposure to bullying develops sensitivity (Herge et al., 2015; Fridh et al., 2015).

In the study, it was determined that there was a significant correlation between the average score of cyberbullying sensitivity scale according to the gender of the adolescents $(\mathrm{p} \leq .001)$. Accordingly, female students were determined to be more sensitive than male students. When the literature is examined, it is thought that the problems that adolescents experience with cyberbullying are closely related to main characteristics such as gender, race, and culture (Horzum and Ayas, 2013; Tomé-Fernández et al., 2019). Many studies support these findings of our study. In a study on the students of the public relations department, it was stated that women were more sensitive to cyberbullying than men (Aktan and Cakmak, 2015). Another study on school counselors reported that female participants were much more sensitive to cyberbullying than men (Horzum and Ayas, 2013). This difference in male and female students can be explained by the fact that women are more sensitive, empathetic, helpful, tolerant and responsible than men.

In the study, the control center sub-dimension score average of the adolescents' health perception scale was $16.62 \pm 2.45$, the precision sub-dimension score average was $12.67 \pm 2.75$, the importance of health sub-dimension score average was $10.29 \pm 2.16$, the self-awareness sub-dimension score average was $8.21 \pm 1.79$ and the total health perception points average was $47.81 \pm 4.79$. In this study, the subdimension in which adolescents scored the highest was the 'control center', while the 'self-awareness' score average was the lowest. In one of the restricted studies evaluating the health perception of adolescents, the highest score among the scale subdimensions was 'control center' while the lowest score was 'self-awareness' similar to this study (Kurt, 2019). In a study evaluating the relationship between nursing students' health perception and health improvement behaviors, participants stated to perceive their health as 'good' and have the highest level of belief in 'controlling their health' (Aciksoz et al., 2013). This indicates that students have control over their health, as in our study. Also, another study on nursing students' health perceptions reported that the highest score in the sub-dimensions of health perception was the 'control center' and the lowest was expressed as the mean of 'self-awareness' score (Ozdelikara et al., 2018). Adolescent's high perception of health may have an advantage in increasing positive health behaviors for future years 
(Zaybak and Fadıloglu, 2004; Kurtuncu et al., 2015). In this study, it can be said that adolescents have a good level of health perception.

It was found that there was a negative correlation between the 'precision' sub-dimension of the health perception scale of adolescents and the score averages for their sensitivity to cyberbullying $(p<0.05)$. Accordingly, as the precision on the perception of health decreases, the sensitivity to cyberbullying increases. A study on peer victimization in adolescents in the United States found that peer victimization and cyberbullying are directly and indirectly related to general health perception and somatic complaints (Herge et al., 2015). The limited studies available in the literature support the relationship between health perception and cyberbullying sensitivity in our study. The rapid change of scientific and technological knowledge, in other words, the lack of "precision" in the field of health can increase the cyberbullying sensitivity. That is why the perception of health is a factor that affects the individual's responsibility, behavior and sensitivity (Velderman et al., 2010).

\section{Conclusion}

In this study, which was conducted to determine the relationship between adolescents' health perceptions and their cyberbullying sensitivity and the factors affecting them, it was found that both male and female adolescents had a high sensitivity to cyberbullying, those female students had higher sensitivities about cyberbullying by gender, that their perceptions of health were at a good level, that there was a negative relationship between the subdimension of health perception 'precision' and the sensitivity to cyberbullying.

The increase of studies in different regions and with different samples to demonstrate health perception and sensitivity to cyberbullying, the expansion of the studies to include the school-family and social circle, and the rise of awareness about the good perception of health were recommended.
Ethics Committee Approval: Ethics committee approval was received for this study from Faculty of Nursing Clinical Research Ethics Committee of Atatürk University. Ethics no: 2019-4/8.

Peer-review: Externally peer-reviewed.

Author Contributions: Concept- A.H.; DesignA.H., T.K.; Materials- A.H.; Data Collection and Processing- A.H., T.K; Literature Review- A.H., T.K.; Writing- A.H., T.K.; Critical Review- A.H., T.K.

Conflict of Interest: No conflict of interest was declared by the authors.

Financial Disclosure: The authors declared that this study hasn't received no financial support.

\section{References}

Aciksoz S, Uzun S, Arslan F. Relationship between perceptions of health status and health promotion behaviors in nursing students. Gulhane Medical Journal 2013; 55(3):181-7.

Akbulut Y, Eristi B. Cyberbullying and victimization among Turkish university students. Australasian Journal of Educational Technology 2011; 27(7):1155-70.

Aktan E, Cakmak V. A Research Related to The Calculation Of Sensitivity For Cyber Bullying İn Social Media Among The Students Of Public Relations. Gumushane University e-Journal of Faculty of Communication 2015; 3(2):160-76.

Al-Rahmi WM, Yahaya N, Alamri MM, Aljarboa NA, Kamin YB, Moafa FA. A model of factors affecting cyber bullying behaviors among university students. İEEE Access 2018; 7:297885.

Bademli K, Lok N. Relationship between the health perception and physical activity of individuals. Turkish Journal of Sport and Exercise 2018; 20(3):127-31.

Baldry AC, Sorrentino A, Farrington DP. Cyberbullying and cyber victimization versus parental supervision, monitoring and control of adolescents' online activities. Children and Youth Services Review 2019; 96:302-7.

Bridge EN, Duman N. Examination of susceptibility to cyberbullying in adolescents in terms of demographic variables. Turkish Cypriot Journal of Psychiatry and Psychology 2019; 1(3):158-65.

Diamond JJ, Becker JA, Arenson CA, Chambers CV, Rosenthal MP. Development of a scale to measure adults' perceptions of health: preliminary findings. Journal of Community Psychology 2007; 35(5): 557-61. 
Fridh M, Lindstrom M, Rosvall M. Subjective health complaints in adolescent victims of cyber harassment: moderation through support from parents/friends-a Swedish population-based study. BMC Public Health 2015; 15(1):949.

Herge WM, La Greca AM, Chan SF. Adolescent peer victimization and physical health problems. Journal of Pediatric Psychology 2015; 41(1):1527.

Horzum MB, Ayas T. Exploring guidance counselors' cyberbullying awareness level in terms of various variables. Hacettepe University Journal of Education 2013; 28(28-3):195-205.

Kadioglu H, Yildiz A. Validity and reliability of Turkish version of perception of health scale. Turkiye Klinikleri Journal of Medical Sciences 2012; 32(1):47-53.

Katz I, Lemish D, Cohen R, Arden A. When parents are inconsistent: Parenting style and adolescents' involvement in cyberbullying. Adolescence Journal 2019; 74:1-12.

Kilinc I, Gunduz S. İnvestigation of high school students' cyber sensitivity, internet addiction and humanistic values. Trakya Journal of Education 2017; 7(2): 261-83.

Kurt GD. Health perception of adolescents in apprenticeships and their way of coping with stress. İstanbul: Marmara University Health Sciences İnstitute. 2019.

Kurtuncu M, Uzun M, Ayoglu FN. The effect of adolescent training program on risky health behaviors and health perception. Journal of Higher Education and Science 2015; 5(2):187-95.

Olenik-Shemesh D, Heiman T. Cyberbullying victimization in adolescents as related to body esteem, social support and social self-efficacy. The Journal of Genetic Psychology 2017; 178(1):28-43.

Ozdelikara A, Alkan SA, Mumcu N. Determination of health perception, health anxiety and effecting factors among nursing students. Medical Journal of Bakirkoy 2018; 14(3):275-82.

Shin N, Ahn H. Factors affecting adolescents' involvement in cyberbullying: What divides the $20 \%$ from the $80 \%$ ?. Cyberpsychology, Behavior, and Social Networking 2015; 18(7):393-9.

Tanrikulu T, Kinay H, Aricak OT. Cyberbullying sensibility scale: Validity and reliability study, Trakya Journal of Education 2013; 3(1):38-47.

Tomé-Fernández M, Ortiz-Marcos JM, OlmedoMoreno EM. Educational environments with cultural and religious diversity: A psychometric analysis of the cyberbullying scale. Religions 2019; 10(7):443.
Uludasdemir D, Kucuk S. Cyberbullying experiences of adolescents and parental awareness: Turkish Example. Journal of Pediatric Nursing 2019; 44:84-90.

Uysal I, Duman G, Yazici E, Sahin M. Pre-service teachers' sensibility in cyberbullying and certain psychometric properties of cyberbullying sensibility scale. Ege Journal of Education 2014; 15(1):191-210.

Velderman M, Crone MR, Wiefferink $\mathrm{CH}$, Reijneveld SA. İdentification and management of psychosocial problems among toddlers by preventive child health care professionals. European Journal of Public Health 2010; 20(3):332-8.

WHO. (2016). Strengthening intersectoral collaboration for adolescent health (No. SEACAH-22). World Health Organization.

Yaman E, Sonmez Z. Cyberbullying trends of adolescence. Online Journal of Technology Addiction \& Cyberbullying 2015; 2(1):18-31.

Zaybak A, Fadiloglu C. Determining of the health promotion behaviors of university students and the factors affecting these behaviors. Journal of Ege University School of Nursing 2004; 20(1):71-95. 